\title{
An Unusual Coexistence of Progressive Pseudorheumatoid Dysplasia and Relapsing Polychondritis
}

\author{
Gamze KILIÇ, Erkan KILIÇ, Özgür AKGÜL, Nimet ATAKUL, Salih ÖZGÖÇMEN \\ Department of Physical Medicine and Rehabilitation, Division of Rheumatology, \\ Medical Faculty of Erciyes University, Kayseri, Turkey
}

\begin{abstract}
Progressive pseudorheumatoid dysplasia is a rare hereditary skeletal disease characterized by bone and cartilage dysplasia, progressive arthropathy without sign of systemic or synovial inflammation. Relapsing polychondritis (RP) is a rare autoimmune disease associated with inflammation in cartilaginous and other proteoglycan rich structures. An associated autoimmune and/or hematologic disorder is seen in over $30 \%$ of patients with RP. To our knowledge, coexistence of progressive pseudorheumatoid dysplasia and RP has not been previously reported. In this article, we describe an unusual case of coexisting progressive pseudorheumatoid dysplasia with RP. Physicians should be aware of this possible association.

Keywords: Polyarthritis; progressive pseudorheumatoid dysplasia; relapsing polychondritis.
\end{abstract}

Progressive pseudorheumatoid dysplasia (PPD) is a rare autosomal recessive inherited disease, leading to restricted mobility of multiple peripheral joints, osseous swelling of the interphalangeal (IP) and other major joints and dysplastic abnormalities of spine including irregularity in vertebral end-plates, short pedicles, and platyspondyly. ${ }^{1}$ Clinical symptoms may develop in early childhood. Patients suffer from stiffness/ swelling of multiple joints without signs of systemic or synovial inflammation. PPD is commonly misdiagnosed as "chronic juvenile polyarthritis with Scheuermann disease". It differs from the seronegative polyarticular rheumatoid arthritis (RA) and other spondyloarthropathies by the absence of inflammatory changes and destructive arthropathy, and the presence of dysplastic bony changes. ${ }^{2}$

Relapsing polychondritis (RP) is a rare episodic and progressive inflammatory disease of cartilaginous and proteoglycan rich structures. RP associated abnormalities include particularly the involvement of elastic cartilage of the ear and nose, hyaline cartilage of peripheral joints, and cartilage of tracheobronchial tree. Also, inflammation of other structures such as the eyes, heart, blood vessels, and kidney may be present. ${ }^{3}$ About 30\% of patients with RP have associated autoimmune disorders, such as systemic connective tissue disease, vasculitis or myelodysplastic syndrome..$^{3,4}$

In this article, we report an unusual case of coexisting PPD and RP. To our knowledge, this association in a patient has not been previously reported in the literature.

\section{CASE REPORT}

A 27-year-old male patient with a nine-year history of RP admitted to our Rheumatology Clinic with polyarthritis. His first symptoms began as painless swelling of IP joints of hand at his late 


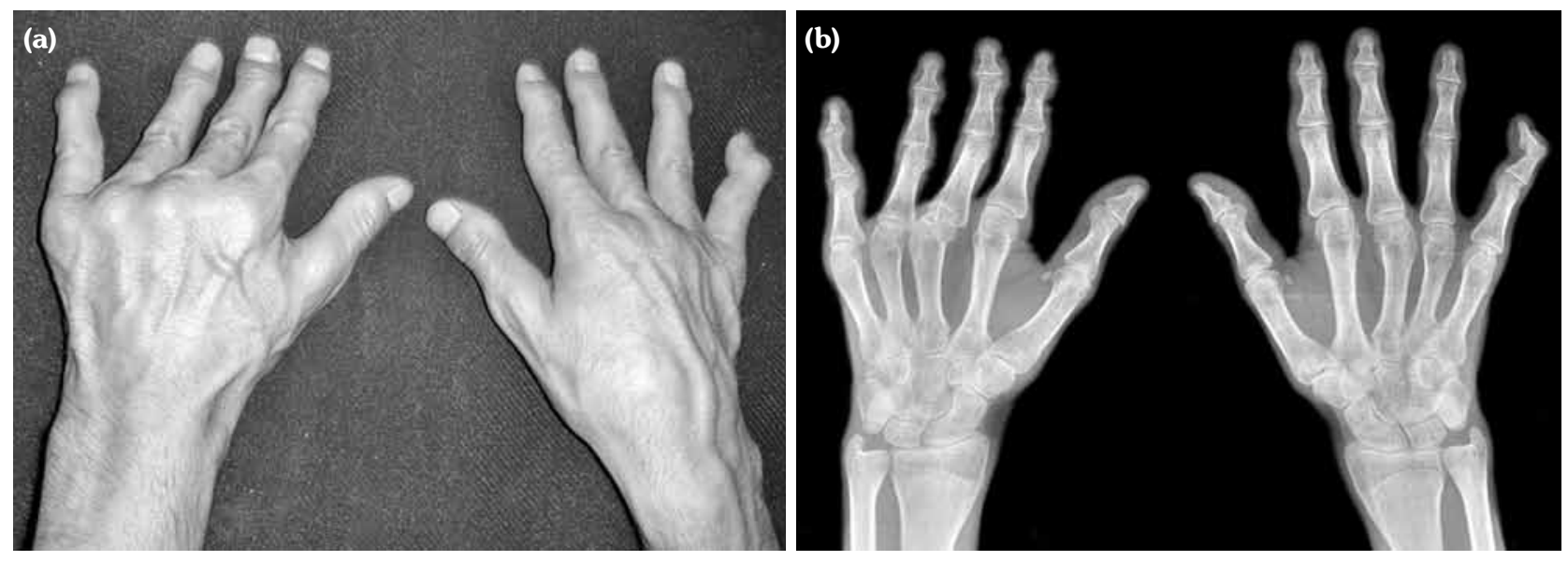

Figure 1. Clinical and radiographic views of hand. (a) Note deformities of metacarpophalangeal and interphalangeal joints on both sides. (b) Hand radiographs show moderate enlargement of metaphyses, squaring of distal epiphysis at level of metacarpophalangeal joints, and narrowing of joint spaces.

childhood. During the subsequent years, joint mobility decreased and spread to other joints, including bilateral wrist, hip, ankle, and foot. He developed painful deformities particularly in his hand and foot joints. Also, he had recurrent episodes of inflammation on his ears and nose (he declared at least three clinically evident episodes). His symptoms related to RP were in remission for the last four years. He had a family history of consanguineous marriage and similar complaints were affecting his sister who had restricted movements and deformities in several joints (particularly hand, wrist, elbow and hip joints). Total hip replacement was performed on her right hip joint due to pain, walking difficulty, and restricted joint motions at age of 28 . We were unable to examine his sister, since she refused to visit our hospital due to social problems. A written informed consent was obtained from the patient.

Physical examination revealed decreased range of motion in shoulders, wrists, metacarpophalangeal, proximal IP, distal IP, and ankle joints. In particular, the IP, metacarpophalangeal and metatarsophalangeal joints had bony enlargements, mild tenderness and flexion contractures; however, no signs of inflammation like redness, effusion, or other signs of synovitis (Figure1). The patient also had mild spinal scoliosis, significant thoracolumbar kyphosis, and pectus excavatum deformity. Furthermore, he had saddle nose deformity, bilateral floppy pinna and audiovestibular dysfunction (sensorineural hearing loss and tinnitus) (Figure 2). The rest of the systemic examination was unremarkable.

Laboratory testing was as follows; erythrocyte sedimentation rate: $2 \mathrm{~mm} /$ hour, C-reactive protein: $4.3 \mathrm{mg} / \mathrm{dL}$ (normal: $0-6 \mathrm{mg} / \mathrm{dL}$ ), leukocyte count: $7.4 \times 10^{3} / \mu \mathrm{L}$, hemoglobin: $16.7 \mathrm{~g} / \mathrm{dL}$, while rheumatoid factor, anti-double stranded deoxyribonucleic acid, antinuclear antibodies, anti-cyclic citrullinated peptide, and brucella agglutination tests were all negative. Urine analysis and liver/renal function tests were normal. Auricular biopsy revealed a loss of basophilic staining, disruption of cartilage integrity, and fibrosis.

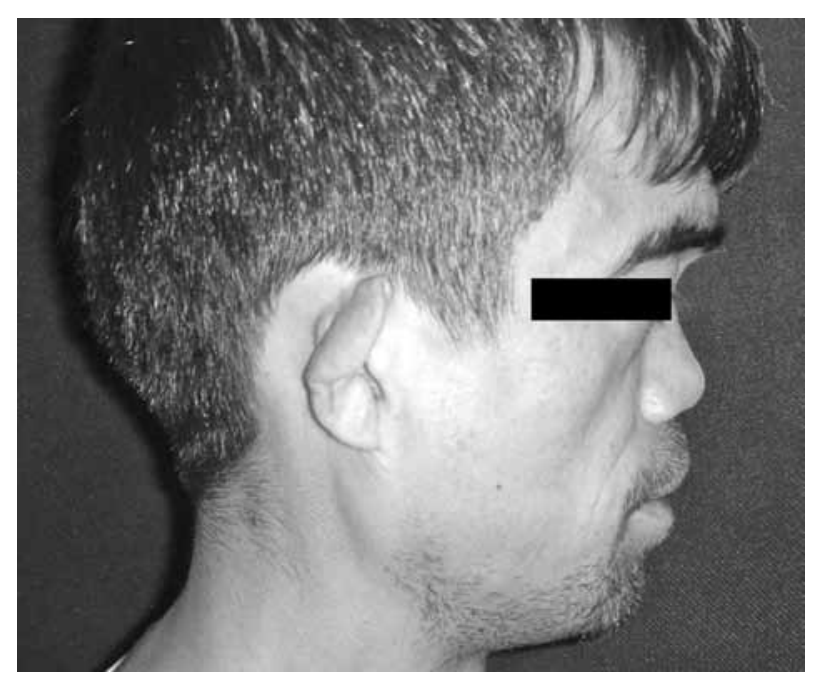

Figure 2. Photograph shows saddle nose deformity and floppy pinna of patient. 


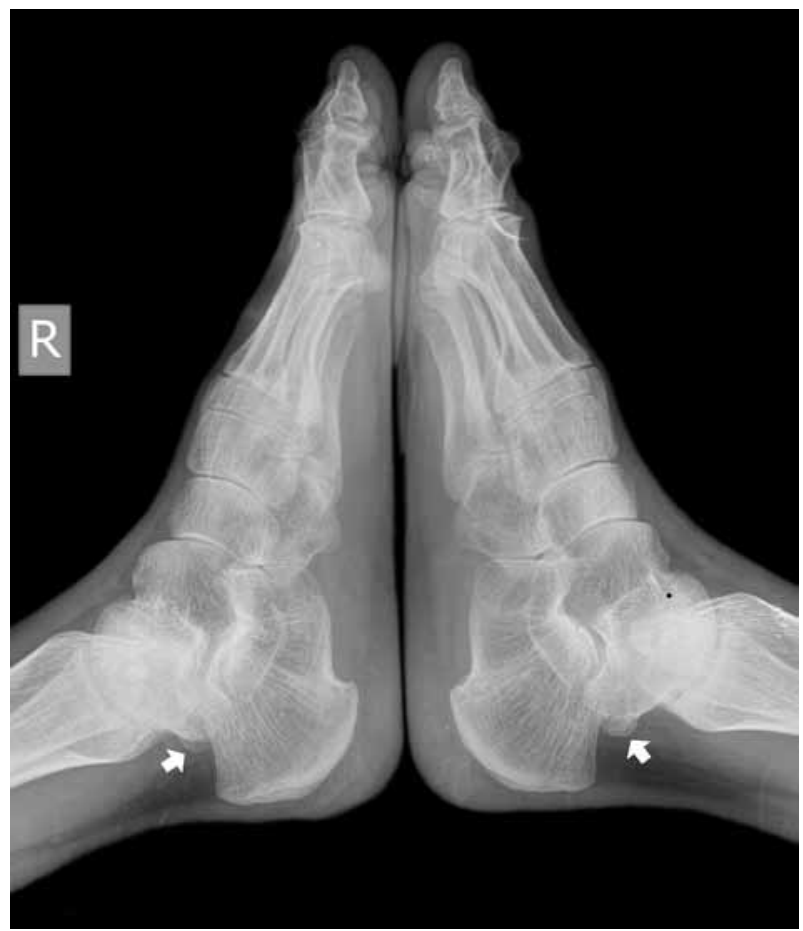

Figure 3. Lateral radiograph of foot indicates mega os trigonum on both sides (arrows).

Radiographs of the hands showed enlargement of metaphyses, flattening of epiphyses, and narrowing of joint spaces with no erosions. Lateral radiograph of the foot revealed bilateral large ossified trigonum attached to the talus (mega os trigonum) (Figure 3). Radiographs of the spine showed increased kyphosis, generalized platyspondyly particularly at the thoracolumbar junction, and irregularity of the vertebral endplates (Figure 4). Magnetic resonance imaging of thoracolumbar spine demonstrated irregularity in vertebral end-plates, multiple disk herniations, and platyspondyly (Figure 5). Dynamic contrastenhanced magnetic resonance imaging of the wrist did not reveal any signs of synovial inflammation.

Based on the clinical and imaging evaluations, the patient was diagnosed as PPD coexisting with RP. He was treated with non-steroid antiinflammatory drugs, physical therapy, and rehabilitation interventions. He was also informed about the disease course, joint protection, and therapeutic exercises.

\section{DISCUSSION}

Progressive pseudorheumatoid dysplasia is a rare inherited skeletal disease characterized by generalized bone/cartilage dysplasia, abnormalities in the vertebrae, and progressive arthropathy without systemic/synovial inflammation. ${ }^{5,6}$ It differs from RA by the absence of synovitis, pannus and other inflammatory changes,

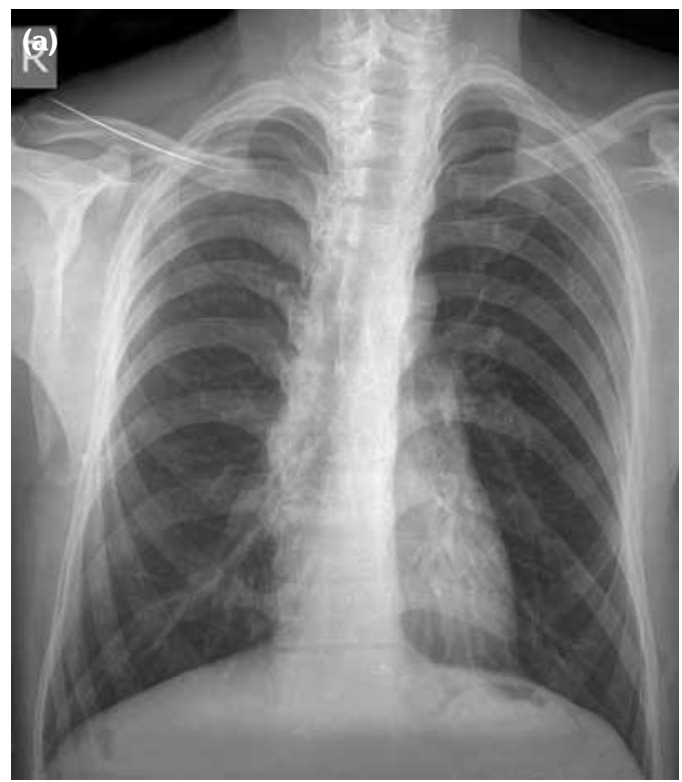

Figure 4. Anteroposterior (a) and lateral views (b) of thoracic spine show platyspondyly, loss of height in vertebral bodies, and kyphoscoliosis.

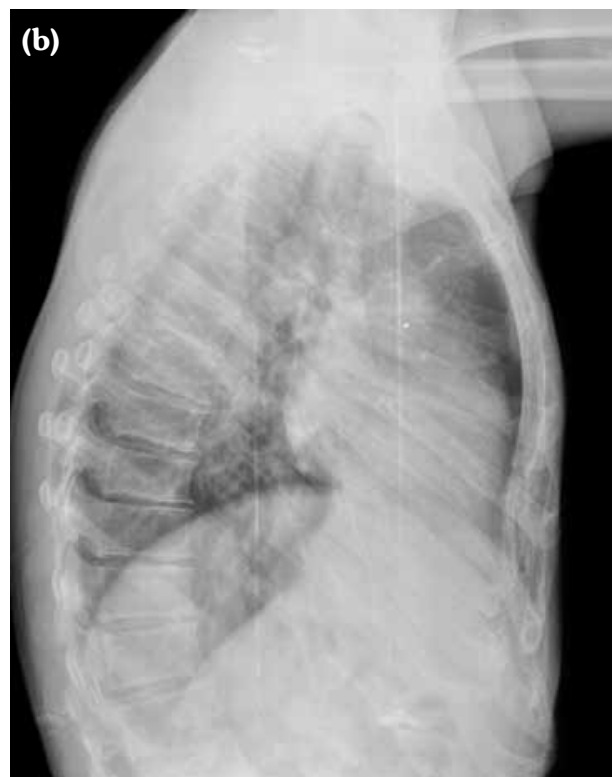



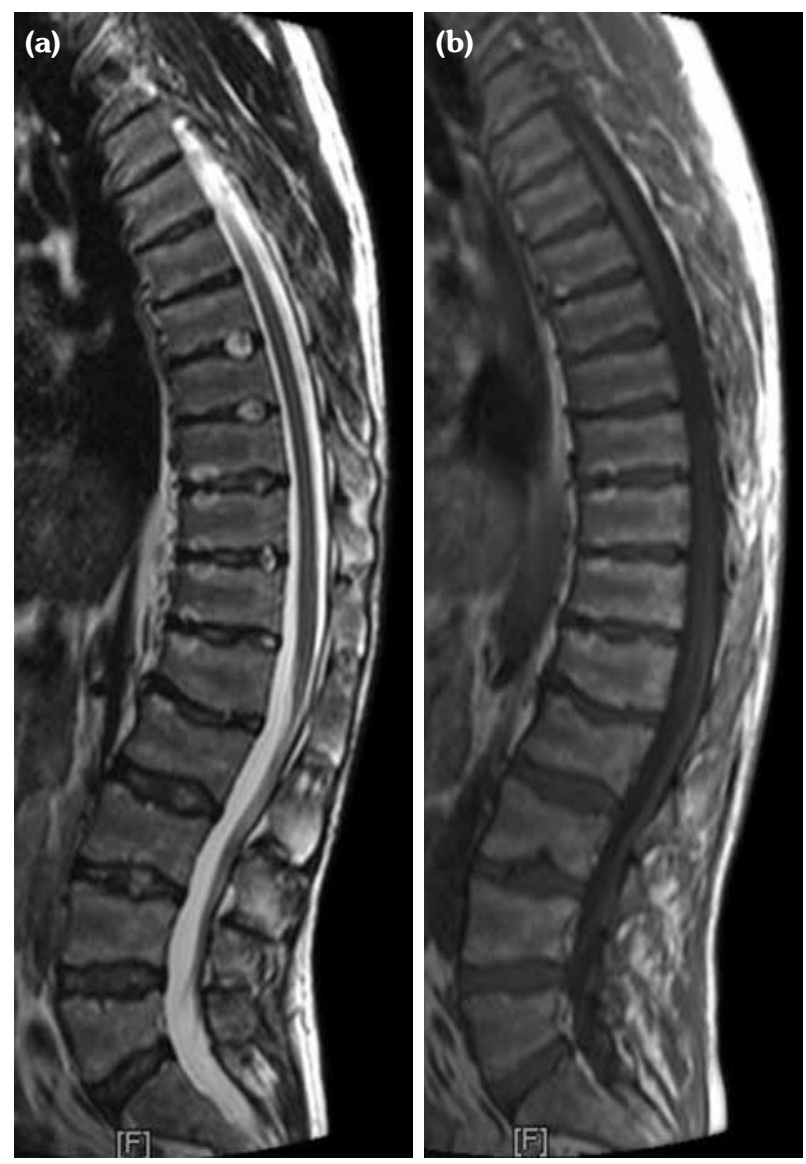

Figure 5. $\mathrm{T}_{2}$ (a) and $\mathrm{T}_{1}$-weighted (b) sagittal magnetic resonance images demonstrate end-plate irregularities, platysphondyly, and multiple intravertebral disk herniations.

and existence of dysplastic bony changes on radiographs. It also does not respond to usual anti-rheumatic treatments. ${ }^{1}$ Although not a sine qua non for diagnosis, genetic testing could be performed in PPD. Mutations in the Wnt1inducible signaling pathway protein 3/(WISP3) gene is found to be associated with PPD. ${ }^{7}$ WISP3 gene encodes a member of the connective tissue growth factor family known to be mostly the extracellular matrix-associated proteins related with regulation of cell migration, proliferation, and differentiation. ${ }^{8,9}$ In our patient, we were unable to perform this genetic testing because of the cost, yet our clinical and imaging features were sufficient for clear diagnosis.

Clinical features of PPD have been described in several reports. ${ }^{9-11}$ The radiographic features include varying degrees of epiphyseal involvement with enlargement of the epi-metaphyseal region, progressive joint narrowing, generalized platyspondyly, multiple intervertebral herniations, kyphoscoliosis, mega os trigonum and bone destruction with aging. ${ }^{2,6,10}$ Our patient had progressive restriction of several joints, IP enlargements, dysplastic bone/cartilage changes, mega os trigonum and platyspondyly, with normal acute phase reactants. The presence of dysmorphic features and the absence of inflammatory signs should alert the physicians for the possible hereditary dysplasias like PPD.

Relapsing polychondritis is a rare autoimmune disorder of unknown etiology, characterized by episodic inflammation/destruction of cartilaginous structures including the ear, nose, peripheral joints and tracheobronchial tree.,12 Immune damage may also spread to non-cartilaginous tissues like kidney, blood vessels, eye, and heart. Although its prevalence is unknown, incidence is reported at 3.5 cases per million. ${ }^{12}$ Over $30 \%$ of patients with RP have an associated systemic disorder, usually an autoimmune/hematologic disorder including systemic vasculitis, RA, systemic lupus erythematosus, or Sjögren's syndrome. ${ }^{3,13,14}$ Our case is interesting since coexisting RP and PPD in the same patient has not been previously reported in the literature.

Articular manifestations may occur in 70\% to $80 \%$ of patients with RP. The most frequent pattern is migratory, seronegative, episodic, and asymmetric inflammatory oligo/polyarthritis. Articular lesions are generally non-erosive/nondestructive, unless the presence of underlying RA. Arthritis associated with RP may affect all synovial joints; however, metacarpophalangeal, IP and knee are the most commonly affected joints. Plain radiographs typically illustrate mild joint space narrowing and juxtaarticular osteoporosis. In patients with RP, cartilage damage may also lead to pectus excavatum deformity. ${ }^{15}$ Despite occurrence of seronegative, non-erosive, noninflammatory polyarthritis, RP does not cause abnormalities in vertebrae, dysplastic bony changes, and progressive arthropathy. However, presences of chronic polyarthritis and progressive loss of joint motions with enlargement of the metacarpal and phalangeal epiphyses, mega os trigonum and spinal abnormalities including platyspondyly make our case an exceptional and interesting one. 
In conclusion, we would like to indicate that a combination of polyarthritis and dysplastic abnormalities of the spine and bony changes without systemic/synovial inflammation should alert clinicians for a possible diagnosis of rare inherited diseases like PPD. Furthermore, with this case report, PPD should be added to the list of diseases potentially coexisting with RP.

\section{Declaration of conflicting interests}

The authors declared no conflicts of interest with respect to the authorship and/or publication of this article.

\section{Funding}

The authors received no financial support for the research and/or authorship of this article.

\section{REFERENCES}

1. Mampaey S, Vanhoenacker F, Boven K, Van Hul W, De Schepper A. Progressive pseudorheumatoid dysplasia. Eur Radiol 2000;10:1832-5.

2. Spranger J, Albert C, Schilling F, Bartsocas C, Stöss H. Progressive pseudorheumatoid arthritis of childhood (PPAC). A hereditary disorder simulating rheumatoid arthritis. Eur J Pediatr 1983;140:34-40.

3. Letko E, Zafirakis P, Baltatzis S, Voudouri A, LivirRallatos C, Foster CS. Relapsing polychondritis: a clinical review. Semin Arthritis Rheum 2002;31:384-95.

4. Mathew SD, Battafarano DF, Morris MJ. Relapsing polychondritis in the Department of Defense population and review of the literature. Semin Arthritis Rheum 2012;42:70-83.

5. Teebi AS, Al Awadi SA. Spondyloepiphyseal dysplasia tarda with progressive arthropathy: a rare disorder frequently diagnosed among Arabs. J Med Genet 1986;23:189-91.

6. el-Shanti HE, Omari HZ, Qubain HI. Progressive pseudorheumatoid dysplasia: report of a family and review. J Med Genet 1997;34:559-63.

7. Temiz F, Ozbek MN, Kotan D, Sangun O, Mungan NO, Yuksel B, et al. A homozygous recurring mutation in WISP3 causing progressive pseudorheumatoid arthropathy. J Pediatr Endocrinol Metab 2011;24:105-8.

8. Sen M, Cheng YH, Goldring MB, Lotz MK, Carson DA. WISP3-dependent regulation of type II collagen and aggrecan production in chondrocytes. Arthritis Rheum 2004;50:488-97.

9. Ehl S, Uhl M, Berner R, Bonafé L, Superti-Furga A, Kirchhoff A. Clinical, radiographic, and genetic diagnosis of progressive pseudorheumatoid dysplasia in a patient with severe polyarthropathy. Rheumatol Int 2004;24:53-6.

10. Kaibara N, Takagishi K, Katsuki I, Eguchi M, Masumi S, Nishio A. Spondyloepiphyseal dysplasia tarda with progressive arthropathy. Skeletal Radiol 1983;10:13-6.

11. Kaya A, Ozgocmen S, Kiris A, Ciftci I. Clinical and radiological diagnosis of progressive pseudorheumatoid dysplasia in two sisters with severe polyarthropathy. Clin Rheumatol 2005;24:560-4.

12. Kent PD, Michet CJ Jr, Luthra HS. Relapsing polychondritis. Curr Opin Rheumatol 2004;16:56-61.

13. Pazirandeh M, Ziran BH, Khandelwal BK, Reynolds TL, Khan MA. Relapsing polychondritis and spondyloarthropathies. J Rheumatol 1988;15:630-2.

14. Gergely P Jr, Poór G. Relapsing polychondritis. Best Pract Res Clin Rheumatol 2004;18:723-38.

15. Knipp S, Bier H, Horneff G, Specker C, Schuster A, Schroten $\mathrm{H}$, et al. Relapsing polychondritis in childhood--case report and short review. Rheumatol Int 2000;19:231-4 